\title{
Preceptoria de graduação em enfermagem: uma discussão da enfermagem
}

\section{Undergraduate preceptorship in nursing: a discussion of nursing}

DOI: $10.46919 / \operatorname{archv} 1 n 5-007$

Recebimento dos originais: 10/07/2020

Aceitação para publicação: 30/08/2020

\section{Emanuel Pereira dos Santos}

Mestre em Enfermagem pela UNIRIO (PPGENF/UNIRIO)

Instituição: Hospital Universitário Gaffrée e Guinle/UNIRIO

Rua Mariz e Barros, 775 - Maracanã, Rio de Janeiro - RJ, Brasil

E-mail: emanuelgranarcanjo@gmail.com

\section{Maria José Pessanha Maciel}

Especialista em terapia intensiva pediatrica e neonatal pela Faculdade UNYLEYA

Instituição: Hospital Universitário Gaffrée e Guinle/UNIRIO

Rua Mariz e Barros, 775 - Maracanã, Rio de Janeiro - RJ, Brasil

E-mail: mjopessanha@gmail.com

\section{Luciana Oliveira Simões}

Especialista em Enfermagem Pediátricpela Fundação Oswaldo Cruz (FIOCRUZ)

Instituição: Hospital Universitário Gaffrée e Guinle/UNIRIO

Rua Mariz e Barros, 775 - Maracanã, Rio de Janeiro - RJ, Brasil

E-mail: lucianaoliveirasimoes@gmail.com

\section{Catia Rustichelli Mourão}

Especialista em Enfermagem do Trabalho pelo Centro Universitário Augusto Motta

Instituição: Hospital Universitário Gaffrée e Guinle/UNIRIO

Rua Mariz e Barros, 775 - Maracanã, Rio de Janeiro - RJ, Brasil

E-mail: catiarustichelli@gmail.com

\section{Camilla Santos da Silva}

Especialista em Enfermagem Obstétrica da Escola de Enfermagem Anna Nery (UFRJ)

Instituição: Hospital Universitário Gaffrée e Guinle/UNIRIO

Rua Mariz e Barros, 775 - Maracanã, Rio de Janeiro - RJ, Brasil

E-mail: camilla.silva@unirio.br

\section{Vanessa Silva de Oliveira}

Mestre em Enfermagem pela UERJ (PPGENF/UERJ)

Instituição: Hospital Universitário Gaffrée e Guinle/UNIRIO

Rua Mariz e Barros, 775 - Maracanã, Rio de Janeiro - RJ, Brasil

E-mail: va_oliveira26@hotmail.com 


\title{
RESUMO
}

Esse trabalho se refere a uma discussão sobre a Preceptoria de Graduação em Enfermagem dentro de um Hospital Universitário na Cidade do Rio de Janeiro realizado pelos autores nos setores de MaternoInfantil. O hospital em questão recebe acadêmicos de sua Universidade e os orienta nas atividades gerenciais e assistenciais. Alguns dos profissionais de Enfermagem já se envolvem em eventos e trabalhos para publicação com os acadêmicos da instituição. Método: Esse estudo é uma discussão sobre tópicos e a importância do trabalho da Enfermagem na Preceptoria. Objeto: A discussão sobre a relação de preceptoria de Graduação em Enfermagem dentro do Hospital Universitário. Objetivo: Discutir sobre a importância da preceptoria de Graduação em Enfermagem. Resultados: A interação entre profissionais e acadêmicos de Enfermagem possibilita o crescimento da profissão desde a sua base. Essa possibilidade facilita a atualização dos profissionais, pois se encontram trocando idéias com a academia científica e recebendo atualizações constantes. Conclusão: A interação entre as pessoas que iniciaram a caminhada no processo de Ensino em Enfermagem e os profissionais que se encontram na assistência e na gerência possibilita o crescimento de ambos, contudo, vale ressaltar a busca de melhorias na definição e no investimento ao preceptor.

Palavras-chave: Preceptoria, Educação, Enfermagem Materno-Infantil.

\begin{abstract}
This work refers to a discussion about the Nursing Undergraduate Preceptorship within a University Hospital in the City of Rio de Janeiro carried out by the authors in the Maternal and Child sectors. The hospital in question receives academics from its University and guides them in management and assistance activities. Some of the nursing professionals are already involved in events and works for publication with the academics of the institution. Method: This study is a discussion on topics and the importance of Nursing work in Preceptorship. Object: The discussion about the relationship between the preceptorship of Undergraduate Nursing in the University Hospital. Objective: To discuss the importance of undergraduate nursing preceptorship. Results: The interaction between nursing professionals and academics enables the profession to grow from its base. This possibility facilitates the updating of professionals, as they are exchanging ideas with the scientific academy and receiving constant updates. Conclusion: The interaction between the people who started the journey in the Nursing Teaching process and the professionals who are in care and management enables the growth of both, however, it is worth mentioning the search for improvements in the definition and investment in the preceptor.
\end{abstract}

Keywords: Preceptorship, Education, Maternal-Child Nursing.

\section{INTRODUÇÃO}

Dados da literatura científica mostram que é de suma importância que a qualidade da relação acadêmico/profissional reflete diretamente na qualidade da formação desses indivíduos que vão entrar para o mercado de trabalho. Desse modo, a voz dos enfermeiros/preceptores faz com que o aluno se aproxime do campo e o enfermeiro - o hospital - contribuiu para elucidar alguns problemas referentes às práticas dos acadêmicos em campo (Carvalho, 2008).

Esse texto se refere à discussão sobre a preceptoria na Graduação em Enfermagem referente aos setores Materno-Infantil, sendo mais especifico os setores de Obstetrícia e Enfermaria de Pediatria. Essa discussão sinaliza a interação desses Enfermeiros com os acadêmicos. As interações ocorrem entre a 
assistência, a gerência, trabalhos e eventos que participam e trabalhos preparados para publicação em conjunto para o crescimento do nome da instituição e melhoria da interação acadêmica.

De acordo com Rodrigues (2014):

\begin{abstract}
"Como limitação do estudo, colocou-se a não abrangência aos demais estados do país, realidades que repercutem no contexto dos serviços de saúde. Tal fato sugere o desenvolvimento de novas pesquisas sobre o tema, reflexões coletivas, envolvendo docentes, discentes e preceptores na busca da formação em Enfermagem para a perspectiva do cuidado integral” Rodrigues (2014).
\end{abstract}

A importância de se propagar estudos sobre o assunto aparece ao se perceber que tal prática é contínua e deve ser melhorada para o bem estar da relação acadêmico/profissional. Uma relação saudável permite uma aprendizagem melhor por parte dos acadêmicos e um grande estímulo para atualizações e inserção em outros cursos por parte dos profissionais. Com isso, o serviço de Enfermagem melhora e proporciona o crescimento da profissão.

\title{
2 MÉTODO
}

Esse estudo é uma discussão sobre tópicos e a importância do trabalho da Enfermagem na Preceptoria junto de literatura encontrada de revistas científicas. Essa discussão concerne sobre mostrar quão importante para o lado acadêmico receber a orientação dos profissionais de como se ocorre a prática que eles estão estudando.

Do lado profissional, isso se torna importante em mostrar o conhecimento de quem está na prática. Com essa interação, é possível que os profissionais da prática sejam estimulados a se atualizar. O questionamento acadêmico permite aos profissionais a oportunidade de estudar para uma atualização e se interessar em entrar em novos cursos.

\section{OBJETO}

Como objeto desse estudo se apresenta a discussão sobre a relação de preceptoria de Graduação em Enfermagem dentro do Hospital Universitário.

\section{OBJETIVO}

Como objetivo desse estudo se apresenta discutir sobre a importância da preceptoria de Graduação em Enfermagem. 


\section{RESULTADOS}

A prática da preceptoria deve ser pautada em planejamentos didáticos e educacionais para que os conhecimentos sejam compartilhados entre a academia e o campo prático. Com isso, o papel do Professor Universitário se faz importante, tanto na sua presença durante a prática, quanto na orientação de melhores abordagens entre acadêmicos e profissionais da prática.

Segundo (Azevedo, 2019) "É importante destacar que a preceptoria, enquanto prática educativa é uma atividade que demanda planejamento, competência, criatividade e sensibilidade”.

Como Hospital Universitário público, recebemos acadêmicos de enfermagem da rede pública de ensino referente à nossa Universidade. Devemos prepará-los para saber interagir de alguma forma com o Sistema Único de Saúde.

Segundo (Azevedo, 2019) “faz-se necessário pensar estratégias que possam qualificar os profissionais para que atuem como preceptores no Sistema Único de Saúde-SUS”.

Um setor público de internação é o campo de atuação dos acadêmicos que os preceptores em questão fazem suas práticas profissionais e educativas. Dados da literatura mostram que a prática em um setor assistencial apresenta fragilidades e limites do ensino no serviço. Isso pode prejudicar a formação da consciência crítica do estudante e o alcance da capacidade de enfrentar e superar os mecanismos de reprodução social do modelo de medicalização e da lógica burocrática da prática da enfermagem (Lima, 2019).

A relação entre os preceptores da prática, os acadêmicos e os professores faz com que se tenha sucesso para uma aprendizagem de qualidade. Segundo Rodrigues (2014) “As relações docente-discenteusuário na integração ensino-serviço desvelaram faces do modo como ocorre a formação em saúde e a compreensão do contexto articulado com as propostas apresentadas pelo SUS”.

Ainda de acordo com Lima (2019):

"Em face destes resultados, destaca-se a necessidade de aprimorar a articulação ensino-serviço na formação da residência e de capacitar pedagogicamente a preceptoria para a problematização da realidade em que elas e as residentes estão inseridas, a fim de que as futuras enfermeiras obstétricas possam contribuir na superação desse modelo e dos fatores limitantes de sua prática profissional nos serviços da atenção obstétrica hospitalar” Lima (2019).

Ainda de acordo com Batista (2016):

\footnotetext{
"O preceptor em enfermagem surge como um novo ator no SUS, atuando na formação de novos profissionais para o desenvolvimento de competências e habilidades dos mesmos, para enfrentar antigos e novos desafios da área, possibilitando a aprendizagem significativa a partir de uma reflexão do serviço construindo práticas inovadoras e transformadoras para viabilizar o SUS, que possui dificuldades em conseguir implantar seus princípios fundamentais em todo seu território desde o seu nascedouro" (Batista, 2016).
} 
A atividade de preceptoria vem sendo praticada em outras profissões além da Enfermagem. Na Enfermagem a preceptoria está em nível de graduação e residência.

Segundo Batista (2016):

"Percebe-se que a atividade preceptoria está mais acentuada e sendo apontada em estudos na formação do recém-graduado em programas de residências multidisciplinares, fomentando a necessidade de aprofundar os conhecimentos a cerca da preceptoria na pré-graduação dos profissionais de saúde, inclusive da formação da enfermagem” Batista (2016).

Um problema identificado é a definição de preceptoria, pois ainda necessita delimitar de forma mais precisa suas funções e quem pode ser definido como preceptor. Muitos locais ainda não definiram o que é o preceptor e qual sua finalidade.

Segundo Carvalho (2008):

"Outro aspecto da problemática levantada pelos preceptores foi a falta de definição da universidade sobre o que se espera do estágio curricular no processo de formação dos alunos, e, consequientemente, o que se espera do preceptor, fazendo com este elabore de forma isolada as suas próprias atribuições" Carvalho (2008).

Ainda segundo Carvalho (2008):

\begin{abstract}
"Para que a integração entre a universidade e os serviços de saúde ocorra de forma mais efetiva é necessário, ainda, que as relações de poder entre esses dois campos sejam melhor explicitadas, de modo que se possa criar canais mais efetivos de trânsito fluente entre eles. O aprofundamento do olhar sobre as delicadas relações entre professores e preceptores - os primeiros representantes da academia que tendem a ver o profissional do serviço como academicamente despreparado; e os segundos vistos como representantes do serviço que, historicamente, têm percebido a academia como "teórica", longe da realidade dos serviços - é fundamental na construção dos veículos de comunicação e integração entre academia e serviço" Carvalho (2008).
\end{abstract}

\title{
6 CONCLUSÃO
}

É importante definir qual o papel do preceptor dentro de uma unidade de internação. Sente-se falta de definir quem pode ser chamado de preceptor e quem não é preceptor, tendo em vista que é uma prática inerente da profissão, porém não uma obrigação do profissional ser um preceptor caso não tenha o desejo de ser ou não se sinta apto.

Ainda sente-se falta de uma orientação direta sobre suas atividades com os acadêmicos. Com isso, nota-se a necessidade de avaliar o perfil de cada grupo que se apresenta em campo de estágio, pois o preceptor passa a conhecer as habilidades e limitações dos mesmos antes de começar as atividades.

Para os preceptores, fica faltando, em alguns casos, investimento mais aprofundado na qualificação do mesmo. Motivar o preceptor ao estudo é de suma importância. Tendo em vista que, para 
uma preceptoria de melhor qualidade, quanto maior a qualificação acadêmica do preceptor, maiores serão as possibilidades de interações com os acadêmicos e com os professores.

\section{REFERÊNCIAS}

- AZEVEDO GM, et al. Preceptoria de Enfermagem em Saúde da Família: definindo sua identidade e relevância para o Sistema Único de Saúde. 2019; Revista Pró-UniverSUS. 10 (1): 166-168.

- BATISTA J. Preceptoria em enfermagem: formação dos Enfermeiros para o SUS. Revista Rede de Cuidados em Saúde. 2016; 10(3): 1-10.

- CARVALHO ESS, FAGUNDES NC. A inserção da preceptoria no curso de graduação em Enfermagem. Revista RENE. 2008; 9 (2): 98-105.

- LIMA GPV, et al. Ensino em serviço de residentes de enfermagem obstétrica na perspectiva da preceptoria. Revista Cogitare. 2019; 24: 1-11.

- RODRIGUES MMA, et al. Preceptoria na perspectiva da integralidade: conversando com Enfermeiros. Revista Gaúcha de Enfermagem. 2014; 35(2): 106-112. 